\title{
Reliability assessment of deteriorating structures using Bayesian updated probability density evolution method (PDEM)
}

$\operatorname{AUTHOR}(S)$ :

FAN, WenLiang; ANG, Alfredo H. -S.; LI, Zhengliang

CITATION:

FAN, WenLiang ... [et al]. Reliability assessment of deteriorating structures using Bayesian updated probability density evolution method (PDEM). Japan-China Workshop on Analysis and Optimization of Large-scale Structures 2018: 3-5: A02.

ISSUE DATE:

2018-05-14

URL:

http://hdl.handle.net/2433/231235

RIGHT: 


\title{
Reliability assessment of deteriorating structures using Bayesian updated probability density evolution method (PDEM)
}

\author{
WenLiang FAN ${ }^{1 *, 2}$, Alfredo H.-S. ANG ${ }^{3}$, ZhengLiang LI ${ }^{1,2}$
}

$1^{*}$ Key Laboratory of New Technology for Construction of Cities in Mountain Area (Chongqing University), Ministry of Education, Chongqing 400045, China

E-mail address: davidfwl@126.com

${ }^{2}$ School of Civil Engineering, Chongqing University, Chongqing 400045, China

${ }^{3}$ Department of Civil and Environmental Engineering, University of California-Irvine, CA 92697, USA

Keywords: reliability, deteriorating structures, Bayesian updating, probability density evolution method, Bayesian updated generalized density, evolution equation

\section{Introduction}

The inspection of engineering structures is important to ensure performance; in this regard, the Bayesian updating process [1] based on inspection data plays an essential role in the life-cycle management of engineering systems. In this paper, the probability density evolution method (PDEM), a universal approach for dynamical systems, is extended for the analysis of deteriorating structures with inspection by combining with the Bayesian updating process [2].

Firstly, the generalized density evolution equation (GDEE) for deteriorating structures is derived. By introducing the deteriorating process $\Theta_{D, i}(t)=\Theta_{D, i, 0} \cdot f_{i}\left(\boldsymbol{\Theta}_{d, D, i}, t\right)$ [3], the response function of a deteriorating structure with time-invariant loads can be written as

$$
Z(t)=g\left[\boldsymbol{\Theta}_{C}, \boldsymbol{\Theta}_{D, 1,0} \cdot f_{1}\left(\boldsymbol{\Theta}_{d, D, 1}, t\right), \cdots, \Theta_{D, s_{2}, 0} \cdot f_{s_{2}}\left(\boldsymbol{\Theta}_{d, D, s_{2}}, t\right), t\right] \triangleq h\left(\boldsymbol{\Theta}_{C}, \boldsymbol{\Theta}_{D, 0}, \boldsymbol{\Theta}_{d, D}, t\right)
$$

With the conditional PDF of $Z(t)$, namely

$$
p_{Z \mid \boldsymbol{\theta}}(z, t \mid \boldsymbol{\theta})=\delta(z-h(\boldsymbol{\theta}, t))
$$

the GDEE [4] can be derived as

$$
\begin{gathered}
\frac{\partial p_{Z \boldsymbol{\Theta}}(z, \boldsymbol{\theta}, t)}{\partial t}+\dot{h}(\boldsymbol{\theta}, t) \frac{\partial p_{Z \boldsymbol{\Theta}}(z, \boldsymbol{\theta}, t)}{\partial z}=0 \\
p_{Z \boldsymbol{\Theta}}\left(z, \boldsymbol{\theta}, t_{0}\right)=p_{\boldsymbol{\Theta}}(\boldsymbol{\theta}) \delta\left(z-h\left(\boldsymbol{\theta}, t_{0}\right)\right) \\
p_{Z}(z, t)=\int_{\Omega_{\boldsymbol{\Theta}}} p_{Z \boldsymbol{\Theta}}(z, \boldsymbol{\theta}, t) \mathrm{d} \boldsymbol{\theta}
\end{gathered}
$$

Secondly, the Bayesian updating for variables with inspection at different time instants is presented based on certain rational but simple assumptions, together with a sampling and fitting approach for the posterior PDF and the predictive one. By using the famous formula for Bayesian updating as follows

$$
p_{\boldsymbol{\Theta}_{U}}^{(p o)}(\boldsymbol{\theta})=\frac{p_{\boldsymbol{\Theta}_{U}}^{(p r)}(\boldsymbol{\theta}) L(\boldsymbol{\theta})}{\int_{\Omega_{\Theta_{U}}} p_{\boldsymbol{\Theta}_{U}}^{(p r)}(\boldsymbol{\theta}) L(\boldsymbol{\theta}) \mathrm{d} \boldsymbol{\theta}}
$$

the time-independent variable $\boldsymbol{\Theta}_{C}$ and the time-dependent variable $\boldsymbol{\Theta}_{D}(t)$ can be updated respectively. With the Bayesian updating, the probabilistic information of variables changes, and a Bayesian updated GDEE (BU-GDEE), which is an impulsive partial differential equation and equivalent to a piecewise ordinary GDEE, is 
developed and proposed for deteriorating structures with inspection. The time-dependent joint PDF of $\boldsymbol{\Theta}$ can be expressed by a Heaviside function as follows

$$
p_{\boldsymbol{\Theta}}(\boldsymbol{\theta}, t)=\sum_{k=1}^{N_{s}} p_{\boldsymbol{\Theta}}^{(k)}(\boldsymbol{\theta}) \cdot\left[H\left(t-t_{k}\right)-H\left(t-t_{k+1}\right)\right]
$$

By combining Eq.(2) with Eq.(7) and according to the basic probability theory, the joint PDF of $Z(t)$ and $\boldsymbol{\Theta}$ is

$$
p_{Z \boldsymbol{\Theta}}(z, \boldsymbol{\theta}, t)=p_{Z \mid \boldsymbol{\Theta}}(z, t \mid \boldsymbol{\theta}) p_{\boldsymbol{\Theta}}(\boldsymbol{\theta}, t)=\delta(z-h(\boldsymbol{\theta}, t)) \cdot \sum_{k=1}^{N_{s}} p_{\boldsymbol{\Theta}}^{(k)}(\boldsymbol{\theta}) \cdot\left[H\left(t-t_{k}\right)-H\left(t-t_{k+1}\right)\right]
$$

Differentiating both sides of Eq.(8) with respect to $t$ will lead to the GDEE of a time-dependent structure with Bayesian updating (BU-GDEE) as follows

$$
\begin{cases}\frac{\partial p_{z \boldsymbol{\Theta}}(z, \boldsymbol{\theta}, t)}{\partial t}+\dot{h}(\boldsymbol{\theta}, t) \frac{\partial p_{z \boldsymbol{\Theta}}(z, \boldsymbol{\theta}, t)}{\partial z}=0, & t \notin T_{I} \\ \Delta p_{z \boldsymbol{\Theta}}\left(z, \boldsymbol{\theta}, t_{k}\right)=\delta\left(z-h\left(\boldsymbol{\theta}, t_{k}\right)\right) \cdot\left\{p_{\boldsymbol{\Theta}}^{(k)}(\boldsymbol{\theta})-p_{\boldsymbol{\Theta}}^{(k-1)}(\boldsymbol{\theta})\right\}, & t_{k} \in T_{I} \\ p_{Z \boldsymbol{\Theta}}\left(z, \boldsymbol{\theta}, t_{0}\right)=\delta\left(z-h\left(\boldsymbol{\theta}, t_{0}\right)\right) \cdot p_{\boldsymbol{\Theta}}^{(0)}(\boldsymbol{\theta}) & \end{cases}
$$

and then

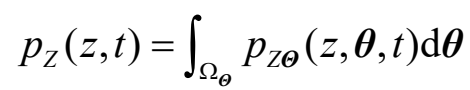

The numerical solution of BU-GDEE based on approximation via a family of $\delta$ sequences [5] is proposed as well. By using the number theoretical method, the representative points of $\boldsymbol{\Theta}$ can be effectively selected. By using the $\delta$ sequences derived from the normal distribution, and introducing the representative points $\boldsymbol{\theta}_{q}^{(k)}$ and their corresponding assigned probability $P_{q}^{(k)}\left(1=1, \cdots, N_{\text {sel }}\right)$, an approximation of Eq.(10) is available. After obtaining the time-dependent PDF of $Z(t)$, the reliability of a time-dependent Bayesian system can be calculated as follows

$$
P_{f}(t)=\operatorname{Pr}\{Z(t)<0\}=\int_{-\infty}^{0} p_{Z}(z, t) \mathrm{d} z
$$

Finally, two numerical examples are analyzed to illustrate the rationale and accuracy of the modified PDEM with Bayesian updating. These examples demonstrate the fact that inspections will highly influence the reliability of deteriorating structures.

\section{Acknowledgements}

The research reported in this paper was conducted under the support of the National Natural Science Foundation of China (Grant Nos. 51678092) and JSPS-NSFC Japan-China Scientific Cooperation Project.

\section{References}

[1] Ang AH-S, Tang WH. Probability concepts in engineering. Emphasis on applications to civil and environmental engineering. 2nd ed. New York: John Wiley \& Sons; 2007.

[2] Zhu B, Frangopol DM. Reliability assessment of ship structures using Bayesian updating. Engineering Structures 2013;56:1836-47.

[3] Enright MP, Frangopol DM. Condition prediction of deteriorating concrete bridges using Bayesian updating. Journal of Structural Engineering 1999;125(10):1118-25.

[4] Li J, Chen J. The principle of preservation of probability and the generalized 
Japan-China Workshop on Analysis and Optimization of Large-scale Structures Supported by JSPS-NSFC Japan-China Scientific Cooperation Project May14, 2018, Kyoto, Japan density evolution equation. Structural Safety 2008;30(1):65-77.

[5] Fan W, Chen J, Li J. Solution of generalized density evolution equation via a family of $\delta$ sequences. Computational Mechanics 2009;43(6):781-96. 